\title{
Les expositions d'origine naturelle en Suisse
}

\author{
CH. MURITH *, A. GURTNER *
}

(Manuscrit reçu le 31 août 1998, accepté le 18 novembre 1998)

RÉSUMÉ L'exposition de la population à la radioactivité d'origine naturelle est une caractéristique permanente et inéluctable de la vie sur terre. Deux sources principales contribuent à cette exposition : les particules de haute énergie du rayonnement cosmique atteignant l'atmosphère terrestre et les nucléides radioactifs provenant de la croûte terrestre, qui marquent notre environnement comme notre propre corps. Ces sources sont responsables de notre exposition externe et interne. Les niveaux de ces expositions et leur domaine de variation en Suisse sont résumés ici et confrontés du point de vue de la dose efficace aux contributions d'origine artificielle. II résulte que les expositions dues au bruit de fond naturel sont plus significatives pour la population que la plupart des expositions issues des sources engendrées par l'homme.

ABSTRACT Exposures to natural radiation in Switzerland.

The exposure of human beings to ionising radiation from natural sources is a continuing and inescapable feature of life on earth. There are two main sources that contribute to this exposure: high-energy cosmic-ray particles incident to the earth's atmosphere and radioactive nuclides that originated in the earth' crust and are present everywhere in the environment, including human body itself. Both external and internal exposures to humans arise from these sources. Exposures to natural radiation sources in Switzerland and some of their variations are here summarised and the resulting effective doses are compared to those from man-made sources exposures. It results that the natural background exposures are more significant for the population than most exposures to man-made sources.

\section{Exposition externe naturelle : (moyenne : $0,8 \mathrm{mSv} / \mathrm{an}$; domaine de variation : 0,5-2 mSv/an)}

L'exposition externe est généralement recensée de manière globale, sans distinction de ses composantes, par des réseaux de mesures télématiques. À l'image de TELERAY en France, la Suisse dispose d'un tel réseau de 58 stations (NADAM) réparties sur l'ensemble du territoire et d'un réseau de 57 stations (MADUK) propre au voisinage des installations nucléaires. L'objectif de ces mesures est de signaler des augmentations artificielles du débit d'exposition. Ce dernier est cependant sujet à des fluctuations d'origine naturelle. Les figures 1 à 3 illustrent certaines de ces variations liées

* Section de surveillance de la radioactivité (SUER), chemin du Musée 3, CH-1700 Fribourg Tél. :+41 263009165 ; Fax :+ 412630097 43; Mél : christophe.murith@bag.admin.ch 
à la pluie, à la neige et, pour une station d'altitude, à une éruption solaire. L'altitude est déterminante pour l'exposition au rayonnement naturel d'origine cosmique. Un vol de ligne de $10 \mathrm{~h}$ à $10000 \mathrm{~m}$ occasionne par exemple pour les passagers une exposition supplémentaire d'environ $0,05 \mathrm{mSv}$. La figure 4 montre les débits d'exposition externe enregistrés avec une chambre d'ionisation lors d'un vol en ballon (Murith, 1987). La mesure sur le lac de Neuchâtel, où la part terrestre est absorbée, permet d'extrapoler la contribution cosmique au niveau du sol. Il est ainsi possible de différencier localement l'exposition au rayonnement cosmique de celle attribuable aux rayonnements gamma des radionucléides primordiaux des séries ${ }^{238} \mathrm{U}$ et ${ }^{232} \mathrm{Th}$ et du ${ }^{40} \mathrm{~K}$, naturellement présents dans la croûte terrestre. Au niveau de la mer, l'exposition externe attribuable au rayonnement cosmique représente $0,27 \mathrm{mSv} / \mathrm{an}$, dont $0,03 \mathrm{mSv} / \mathrm{an}$ de la composante neutronique de ce rayonnement (UNSCEAR, 1993).

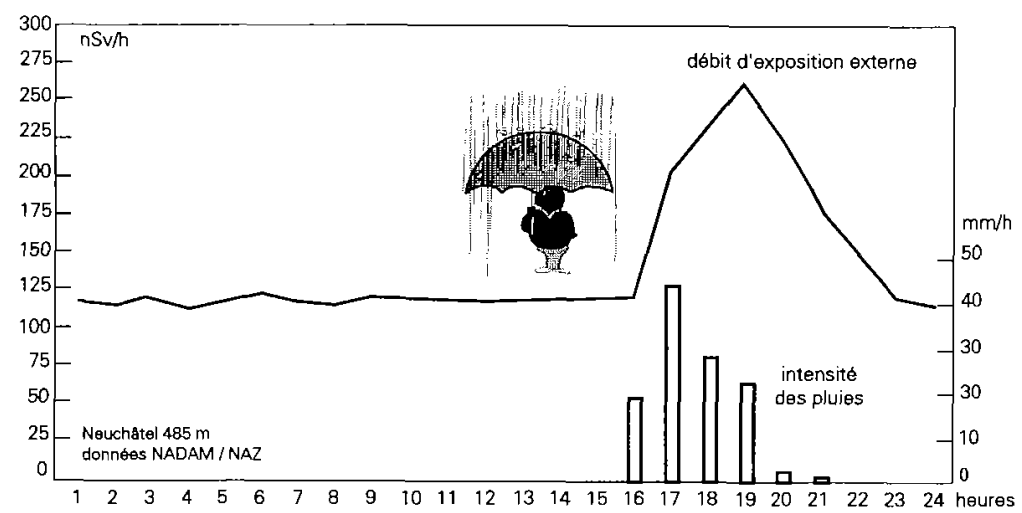

Fig. I. - Influence des précipitations sur l'exposition externe.

Influence of precipitations on external exposure.

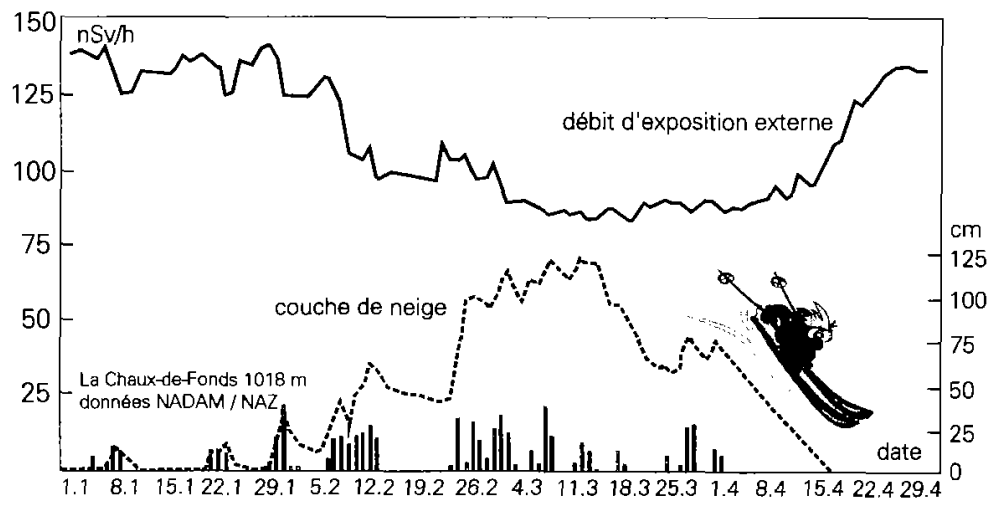

Fig. 2. - Influence de la couverture neigeuse sur l'exposition externe.

Influence of the snow cover on external exposure. 


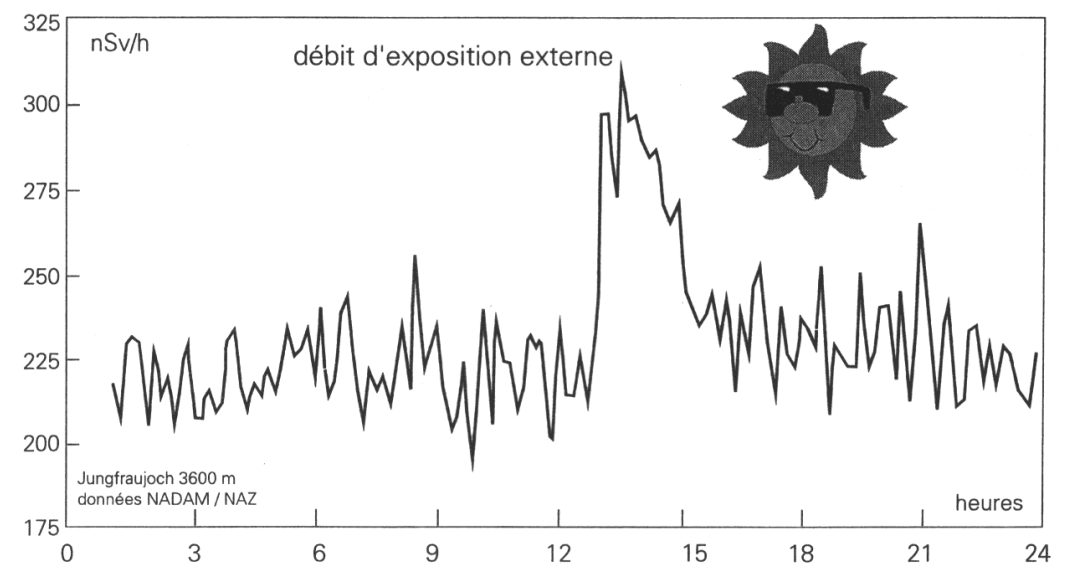

Fig. 3. - Influence d'une éruption solaire sur l'exposition externe.

Influence of a solar eruption on external exposure.
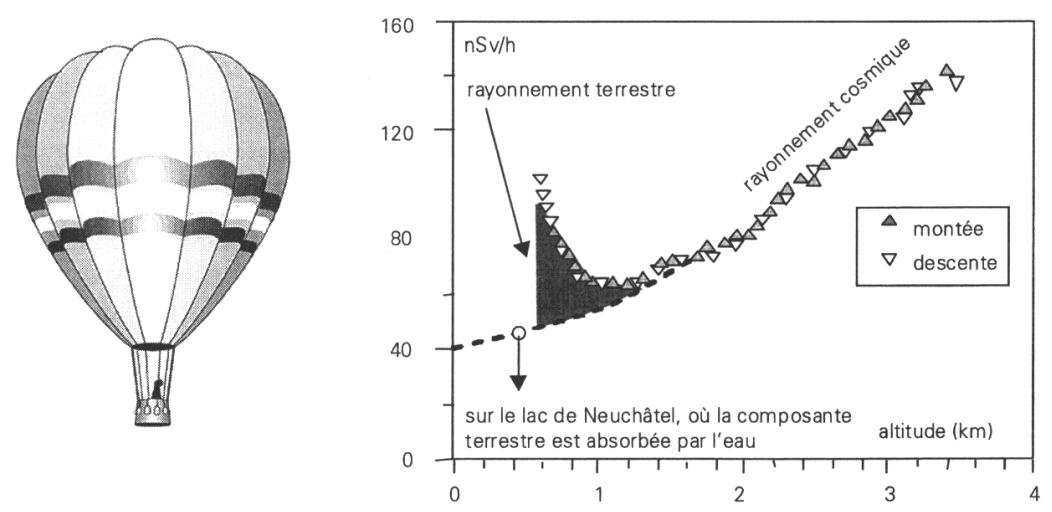

Fig. 4. - Influence de l'altitude sur l'exposition au rayonnement cosmique.

Influence of the altitude on the cosmic-ray exposure.

L'exposition externe attribuable aux radionucléides primordiaux dépend essentiellement des sols et de leur origine géologique. Elle peut être déterminée directement sur site par spectrométrie gamma in situ (ICRU 53, 1994). Cette technique de mesure performante est largement exploitée en Suisse depuis 1984. Ces mesures indiquent en moyenne pour la Suisse les concentrations et les débits d'exposition suivants : ${ }^{238} \mathrm{U}(26 \mathrm{~Bq} / \mathrm{kg}$ et $12 \mathrm{nGy} / \mathrm{h}),{ }^{232} \mathrm{Th}(25 \mathrm{~Bq} / \mathrm{kg}$ et $15 \mathrm{nGy} / \mathrm{h})$, ${ }^{40} \mathrm{~K}(370 \mathrm{~Bq} / \mathrm{kg}$ et $15 \mathrm{nGy} / \mathrm{h})$. Les débits d'exposition sont calculés à $1 \mathrm{~m}$ du sol 
et tiennent compte de tous les descendants supposés en équilibre. En plus de la variabilité géologique, on observe par exemple l'influence de l'utilisation des engrais sur les concentrations de ${ }^{40} \mathrm{~K}$ (jusqu'à $10000 \mathrm{~Bq}{ }^{40} \mathrm{~K} / \mathrm{kg}$ d'engrais) et celle liée au dépôt des descendants du radon par la pluie. La figure 5 résume l'exposition au rayonnement terrestre obtenue par cartographie géostatistique (Allen, 1998) des examens in situ. Cette représentation traduit la variabilité de cette composante sur le territoire suisse et fait ressortir les régions aux niveaux élevés.

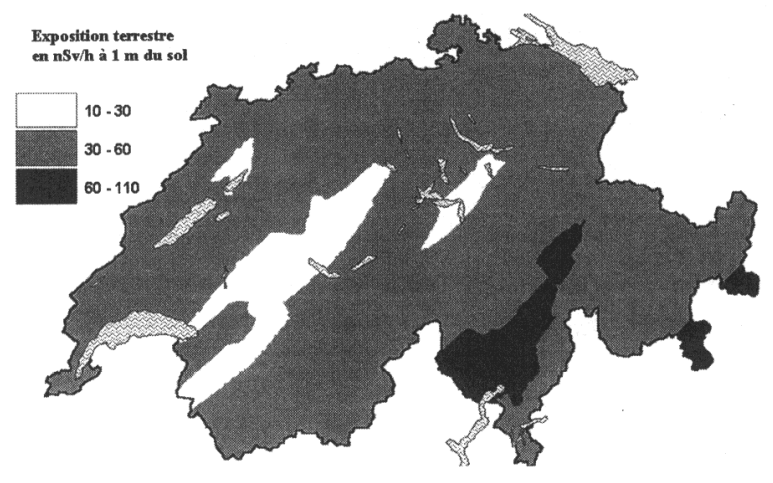

Fig. 5. - Influence des radionucléides primordiaux sur l'exposition externe en plein air.

Influence of the primordial radionuclides on the external exposure.

Les comparaisons avec les mesures de l'exposition externe globale (chambre d'ionisation) atteste de la fiabilité des contributions individuelles calculées à partir des concentrations in situ mesurées dans le sol, et en surface de celui-ci ou dans l'air, en cas de contamination (Murith et Gurtner, 1996). Les principaux avantages de la méthode par rapport aux mesures en laboratoire d'échantillons sont la disponibilité rapide des résultats sur le site et l'obtention de moyennes sur des variations spatiales des sources à faible échelle, qui ne sont en réalité pas relevantes du point de vue de l'exposition externe.

Les matériaux de construction selon leur provenance renferment aussi dans différentes proportions les radionucléides primordiaux. Les comparaisons de l'exposition externe en plein air et dans des maisons à proximité indiquent en Suisse des valeurs en moyenne $10 \%$ plus élevées à l'intérieur qu'à l'extérieur (Fig. 6). Cela semble signifier que l'exposition issue des radionucléides primordiaux dans les matériaux de construction est généralement plus importante que la réduction de la part cosmique due à la structure du bâtiment. Si l'on tient compte d'un séjour plus fréquent à l'intérieur (19 h/jour) qu'à l'extérieur (5 h/jour), l'exposition externe d'origine naturelle en Suisse correspond en moyenne à une dose efficace annuelle de $0,8 \mathrm{mSv} / \mathrm{an}$ pour un domaine de variation s'échelonnant de 0,5 à $2 \mathrm{mSv} / \mathrm{an}$. 


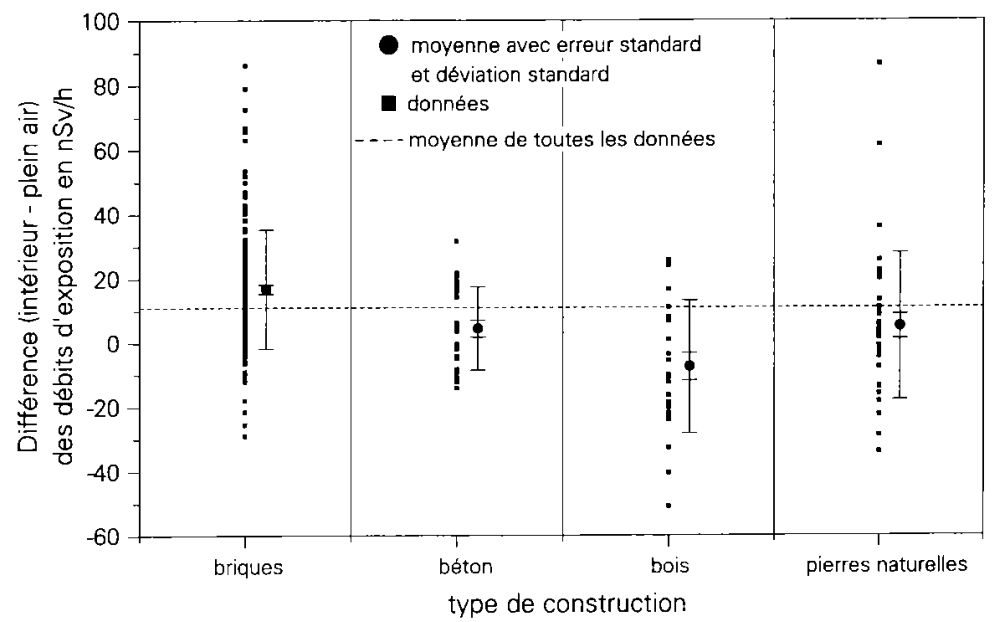

Fig. 6. - Influence des matériaux de construction sur l'exposition externe globale.

Influence of building materials on the global external exposure.

\section{Exposition interne naturelle : (moyenne : $2 \mathrm{mSv} / \mathrm{an}$; domaine de variation : 0,5-100 $\mathrm{mSv} / \mathrm{an}$ )}

Les radionucléides naturels que l'on trouve dans les différents compartiments de l'environnement peuvent aussi être transférés partiellement d'un milieu à l'autre jusqu'à leur assimilation préférentielle dans certains organes et tissus du corps, où ils contribuent à l'exposition interne de l'être humain par inhalation ou par ingestion. Pour l'exposition par inhalation, le milieu atmosphérique est essentiellement contrôlé en Suisse par le réseau d'alerte RADAIR ( 11 moniteurs $\alpha / \beta$ avec compensation naturelle, 3 moniteurs iode et 1 moniteur spectrométrique), et pour les très bas niveaux, par l'analyse en laboratoire des aérosols collectés par les installations à grand débit $\left(500\right.$ à $\left.800 \mathrm{~m}^{3} / \mathrm{h}\right)$. Le réseau RADAIR indique des concentrations naturelles des descendants du radon de 1 à $60 \mathrm{~Bq} / \mathrm{m}^{3}$ selon les sites et les conditions météorologiques. L'analyse gamma des filtres aérosols hebdomadaires montre la présence naturelle dans l'air proche du sol du ${ }^{7} \mathrm{Be}$ cosmogénique $(0,5$ à $\left.8 \mathrm{mBq} / \mathrm{m}^{3}\right)$ et $\mathrm{du}{ }^{210} \mathrm{~Pb}\left(0,2\right.$ à $\left.2 \mathrm{mBq} / \mathrm{m}^{3}\right)$. L'analyse mensuelle des précipitations signale outre le ${ }^{7} \mathrm{Be}(0,1$ à $1,5 \mathrm{~Bq} / 1)$, la présence ${ }^{3} \mathrm{H}$, dont la part naturelle inférieure au $\mathrm{Bq} / \mathrm{l}$, est augmentée par des injections artificielles. L'inhalation des radionucléides naturels autres que le radon et ses descendants ne représente qu'une contribution mineure à l'exposition interne. Par contre, le radon et ses produits de désintégration à vie courte sont responsables de l'exposition prédominante du public aux sources naturelles. En particulier les émetteurs alpha ${ }^{218} \mathrm{Po}_{\mathrm{ot}}{ }^{214} \mathrm{Po}$, inhalés avec l'air, les poussières ou la fumée irradient les voies bronchiques, où se trouvent les 
cellules les plus sensibles au développement d'un cancer du poumon. L'examen de 25000 maisons indique une moyenne géométrique de $60 \mathrm{~Bq}^{222} \mathrm{Rn} / \mathrm{m}^{3} \mathrm{~d}$ 'air. En se basant sur des taux d'occupation de $7000 \mathrm{~h} / \mathrm{an}$ et un facteur de dose de $2,4410^{-6} \mathrm{mSv}$ par Bqh/m $/ \mathrm{m}^{3}$ pour l'habitation, resp. $2000 \mathrm{~h} /$ an et $3,1710^{-6} \mathrm{mSv}$ par $\mathrm{Bqh} / \mathrm{m}^{3}$ pour le lieu de travail, on obtient une dose efficace annuelle due au radon de $1 \mathrm{mSv} / \mathrm{an}$ (habitation) respectivement de $0,4 \mathrm{mSv} / \mathrm{an}$ (travail). Ces valeurs sont encore augmentées d'environ $10 \%$ si l'on tient compte du thoron également naturel. Cela mène en moyenne pour la population suisse à une dose efficace annuelle due au radon et au thoron de l'ordre de 1,6 mSv/an. Environ $90 \%$ des maisons examinées montrent des concentrations radon situées en-dessous de la valeur directrice de $400 \mathrm{~Bq} / \mathrm{m}^{3}$ et $2 \%$ d'entre elles des concentrations supérieures à la limite de $1000 \mathrm{~Bq} / \mathrm{m}^{3}$. En Suisse cette valeur limite est vraisemblablement dépassée dans quelques milliers de maisons. Leur recherche est prioritaire pour la mise en cuvre de mesures d'assainissements appropriées afin d'en réduire l'exposition. Dans le but de mieux cerner les zones à risque, des études de géostatistique sont en cours sur les données disponibles. La figure 7 présente la carte actuelle du radon en Suisse. L'information générale sur le radon en Suisse a fait l'objet d'un cédérom (OFSP, 1995). La concentration en radon la plus élevée dans l'air contenu dans le sol a été mesurée dans le canton des Grisons ( $1000000 \mathrm{~Bq} / \mathrm{m}^{3}$ ). Enfin, pour les amis des cavernes, un jour dans une grotte avec $5000 \mathrm{~Bq} / \mathrm{m}^{3}$ cause environ $0,4 \mathrm{mSv}$.

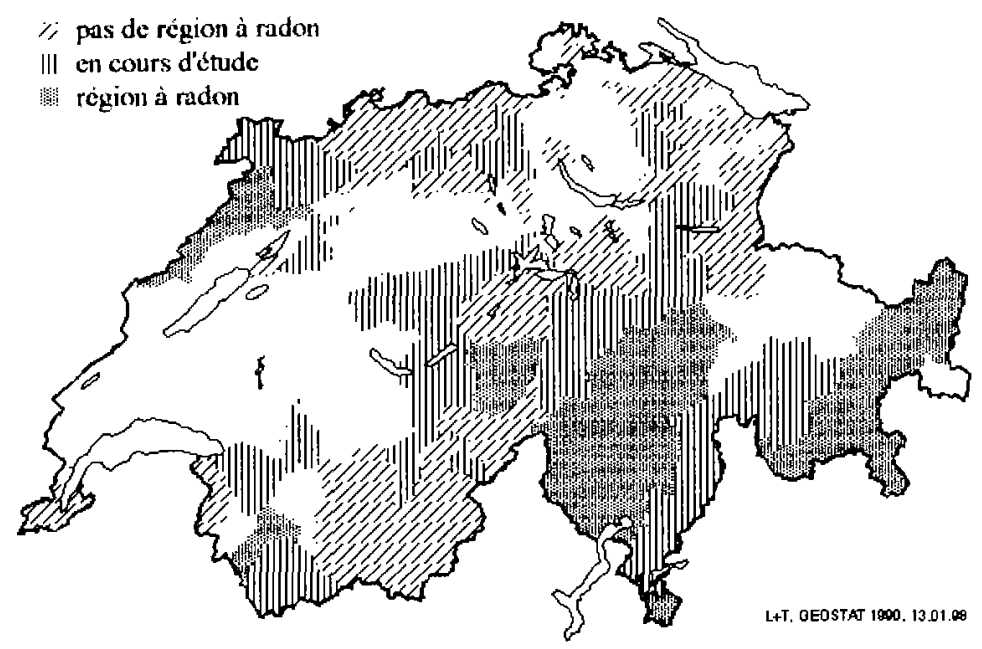

Fig. 7. - Le radon en Suisse. Les districts insuffisamment répertoriés figurent en blanc.

Radon in Switzerland. Districts insufficiently examined appear in white.

Les radionucléides naturels occasionnent également une exposition interne par ingestion suite à leur assimilation dans notre corps avec la nourriture. Les niveaux de cette exposition dépendent largement du type d'aliments, des taux de consommation et des facteurs de dose qui different du nourrisson à l'adulte. Ainsi le ${ }^{210}$ Po présente des 
concentrations relativement élevées dans les produits de la mer. En admettant qu'un adulte consomme annuellement $13 \mathrm{~kg}$ de poissons, $1 \mathrm{~kg}$ de mollusques et $1 \mathrm{~kg}$ de crustacés, il ingère près de $50 \mathrm{~Bq}^{210} \mathrm{Po} / \mathrm{an}$. Compte tenu du facteur de dose élevé du ${ }^{210} \mathrm{Po}\left(1,210^{-6} \mathrm{~Sv} / \mathrm{Bq}\right.$ ingéré$)$, il en résulte une dose efficace de $0,06 \mathrm{mSv} / \mathrm{an}$. En Suisse on estime la contribution naturelle à l'exposition interne par ingestion à environ $0,4 \mathrm{mSv} / \mathrm{an}$ sous l'hypothèse d'un comportement alimentaire habituel. Comme le montre la figure 8 , la contribution majeure provient $\mathrm{du}^{40} \mathrm{~K}$, tandis que les radionucléides cosmogéniques au rôle de traceurs dans l'atmosphère et dans les systèmes hydrologiques occasionnent ensemble des doses efficaces 10 fois moindre. Au niveau des séries ${ }^{238} \mathrm{U}$ et ${ }^{232} \mathrm{Th}$, la consommation régulière d'eaux minérales de teneurs accrues peut aussi entraîner une exposition additionnelle de l'ordre de 0,1 jusqu'à $0,3 \mathrm{mSv} / \mathrm{an}$ (Métivier et Roy, 1997). Des radionucléides naturels sont aussi présents dans d'autres objets courants, tels les carrelages, la poterie et la verrerie $\left({ }^{238} \mathrm{U},{ }^{232} \mathrm{Th}\right)$, les manchons des lampes à incandescence et la céramique dentaire $\left({ }^{232} \mathrm{Th}\right)$, les paratonnerres $\left({ }^{226} \mathrm{Ra}\right)$, des batteries de téléphone mobile $\left({ }^{138} \mathrm{La}\right)$ ou écouteurs de Walkman $\left({ }^{147} \mathrm{Sm}\right)$ et le tabac $\left({ }^{210} \mathrm{Po}\right)$. Leur contribution globale peut être estimée à $0,1 \mathrm{mSv} / \mathrm{an}$.
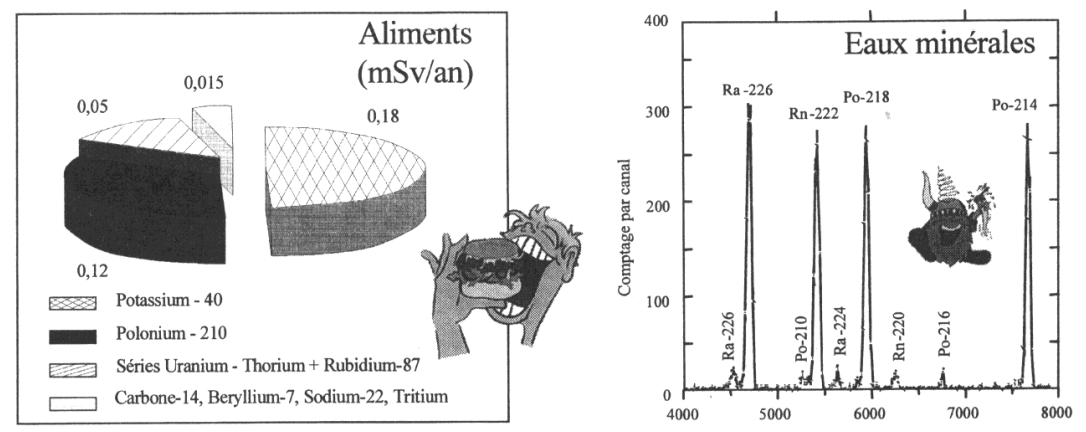

Fig.8. - Influence de l'alimentation sur l'exposition interne.

Influence of foodstuff on the internal exposure.

\section{Augmentations des niveaux naturels dues à la civilisation}

À l'image du radon, qui a de tout temps existé dans l'environnement sans poser de problème connu, mais dont les effets peuvent être nocifs dans des environnements donnés (galeries des mines d'uranium, habitations) issus de la civilisation, le niveau de certains radionucléides naturels a été altéré par l'homme. Ainsi, les niveaux naturels ${ }^{14} \mathrm{C}$ et ${ }^{3} \mathrm{H}$, produits dans l'atmosphère par les interactions des particules cosmiques, ont considérablement augmenté suite aux essais atomiques atmosphériques des années 60, comme le montre la figure 9 (Völkle et al., 1989). 

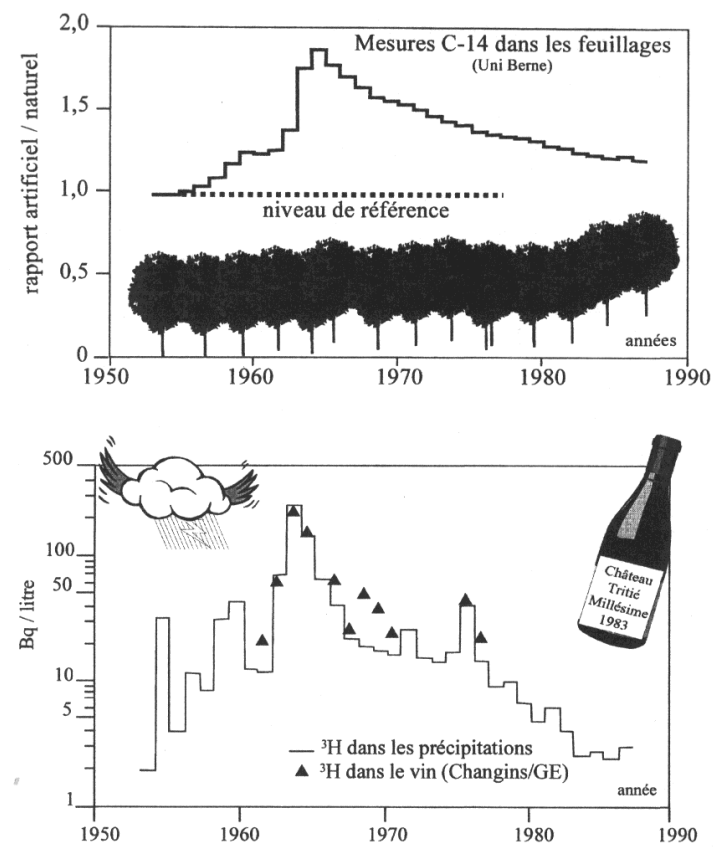

Fig. 9. - Influence des essais atomiques passés sur les niveaux naturels ${ }^{14} \mathrm{C}$ et ${ }^{3} \mathrm{H}$.

Influence of atomic tests on the natural levels of ${ }^{14} \mathrm{C}$ and ${ }^{3} \mathrm{H}$.

À cela s'ajoute les augmentations issues de l'exploitation des centrales nucléaires, des usines de retraitement et de l'industrie. Ainsi par exemple des augmentations d'un facteur 1000 sont décelables pour le ${ }^{3} \mathrm{H}$ dans les précipitations du voisinage immédiat d'industries de peintures luminescentes et jusqu'à $50 \%$ pour ${ }^{1 e}{ }^{14} \mathrm{C}$ dans les feuillages à proximité des fours d'incinération de déchets chimiques. Pour les radionucléides purement artificiels, le ${ }^{85} \mathrm{Kr}$ issu des usines de retraitement de La Hague et Sellafield, montre dans l'air l'activité la plus élevée, de l'ordre de $1 \mathrm{~Bq} / \mathrm{m}^{3}$ en Suisse, mais ne représente qu'une très faible contribution à la dose efficace annuelle d'environ $0,00004 \mathrm{mSv}$. Dans son ensemble l'environnement reste surtout marqué par les activités rémanentes de ${ }^{137} \mathrm{Cs}$ et ${ }^{90} \mathrm{Sr}$ des essais nucléaires et de Tchernobyl comme en témoigne la mémoire des sols (Murith et Gurtner, 1995) et des sédiments (Fig. 10), qui permet de faire l'inventaire des contaminations en lien avec leur origine.

Enfin les mesures directes du corps humain, comme celles du ${ }^{90} \mathrm{Sr}$ dans les dents de lait ou dans les vertèbres humaines (Völkle et al., 1989), du ${ }^{3} \mathrm{H}$ dans l'urine et du ${ }^{137} \mathrm{Cs}$ dans le corps entier servent à vérifier le transfert de la radioactivité de l'environnement vers l'Homme. La figure 11 montre clairement les incorporations liées au ${ }^{137} \mathrm{Cs}$ des essais nucléaires et de Tchernobyl. À titre de comparaison, les valeurs correspondantes du ${ }^{40} \mathrm{~K}$ dans le corps s'échelonnent de 3000 à $5000 \mathrm{~Bq}$. 


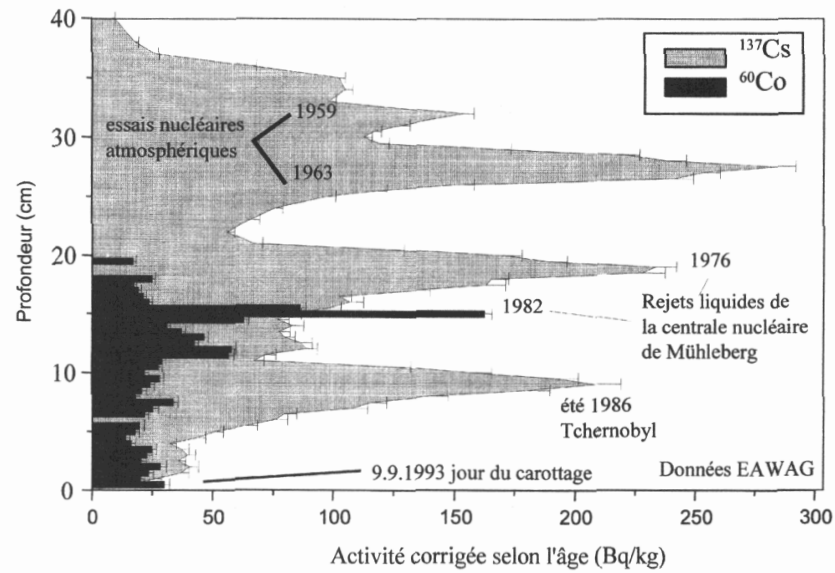

Fig. 10. - Inventaire des contaminations dans un carottage de sédiment du delta de l'Aar. Inventory of contamination's in sediment cores within the Aare delta.

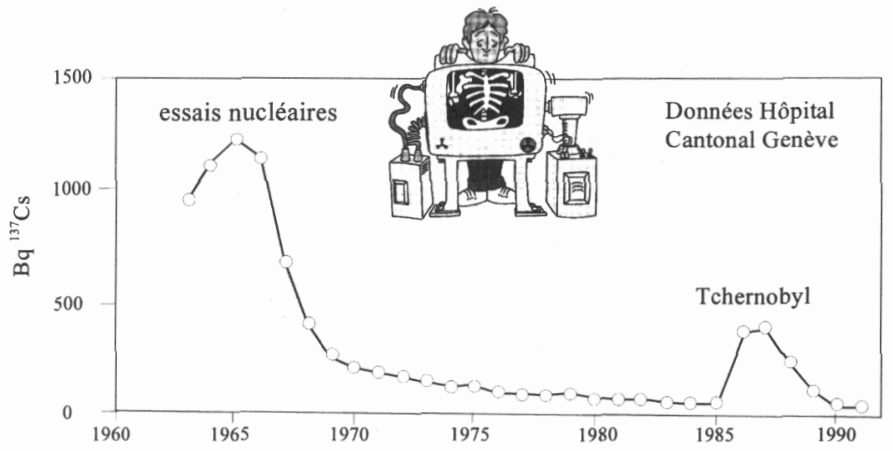

Fig. 11. - Suivi des activités moyennes ${ }^{137} \mathrm{Cs}$ dans le corps d'écoliers de Genève. Mean ${ }^{137} \mathrm{Cs}$ content within student body from Geneva.

\section{Conclusion}

L'exposition moyenne de la population suisse aux sources de rayonnements est voisine de $4 \mathrm{mSv} / \mathrm{an}$. Les contributions naturelles, dont les moyennes et les domaines de variation sont résumés dans la figure 12 dominent. À ces expositions annuelles récurrentes s'ajoutent principalement celles de la médecine (jusqu'à $100 \mathrm{mSv} / \mathrm{an}$, avec en moyenne pour la Suisse $1 \mathrm{mSv} / \mathrm{an})$. Les expositions rémanentes des essais nucléaires et de Tchernobyl ainsi que celles liées à l'industrie et aux installations nucléaires (Murith et Gurtner, 1994) ne représentent actuellement qu'une faible fraction $(<5 \%)$ de l'exposition annuelle moyenne. 


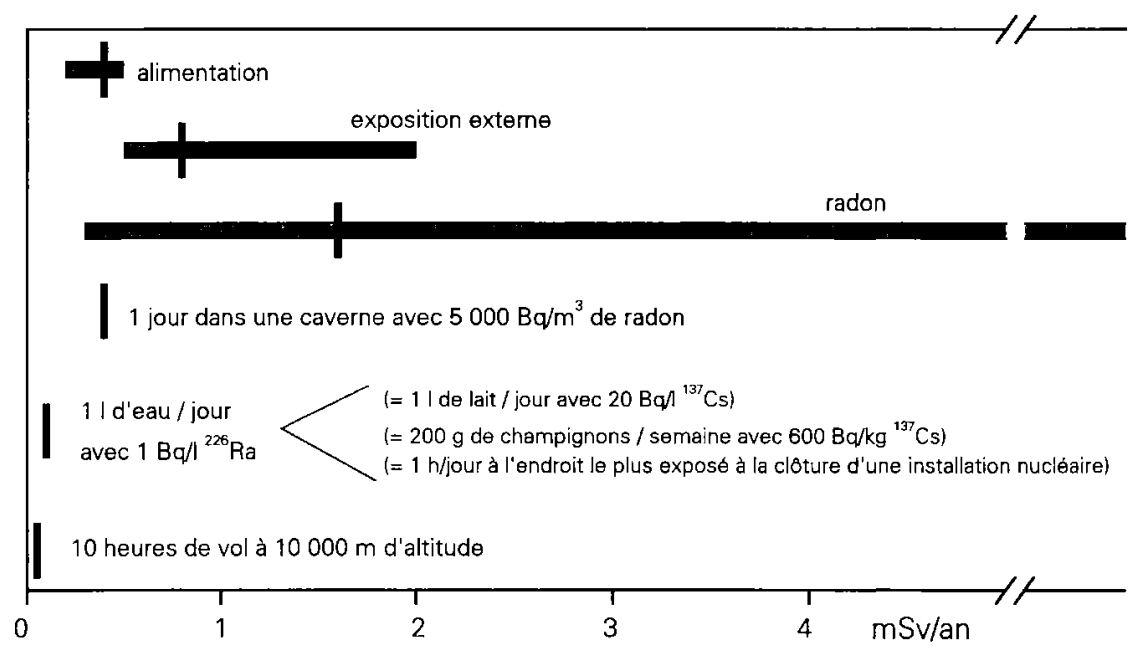

Fig. 12. - Synthèse des expositions naturelles de la population suisse. Natural exposures to the Swiss population.

\section{RÉFÉRENCES}

Allen O. (1998) Innovations méthodologiques et cartographie, Dipl. de géologie et minéralogie, Uni Lausanne.

ICRU (1994) Gamma-Ray Spectrometry in the Environment, ICRU Report 53, Bethesda, U.S.A.

Métivier H.,t Roy M. (1997) Dose efficace liée à la consommation d'eau minérale naturelle par l'adulte et le nourrisson. Radioprotection, 32, 491-499.

Murith C. (1987) La radioactivité de l'environnement : une méthode rapide d'estimation de l'exposition externe du public. Radioprotection, 22, 231-239.

Murith C., Gurtner A. (1994) Radioactivité, Environnement et Centrales nucléaires (1984-1994) ISBN 3-905235-15-3, OFSP.

Murith C., Gurtner A. (1995) Le sol, un témoin de notre patrimoine radioactif. Séminaire concernant l'impact sur l'environnement des radioéléments de longue période, juin 29, 1995, Lucerne, $87-$ 96, ISBN 2-88444-005-4.

Murith C., Gurtner A. (1996) In situ spectrometry to follow the behaviour of the Chernobyl radionuclides in the soil, Symposium. d. Österr. Bodenkundl. Ges., April 22-24, 1996, Vienna, 53, 1926, ISSN 0029-893-X.

OFSP (1995) CD-ROM « Gaston Radon », OCFIM, 3000 Berne, Production : FRY ROM multimédia.

UNSCEAR (1993) Report to the general Assembly with Annexes. United Nations Scientific Committee on the Effects on Atomic Radiation (United Nations, New York).

Völkle H. Murith C., Surbeck H. (1989) Fallout from atmospheric bomb tests and releases from nuclear installations, Radiat. Physics and Chemistry, Special Issue, Radionuclides in the Environment, 34, 261-277. 